\title{
Longevidad y pobreza extrema en Huancavelica, Perú
}

- Úrsula Sandoval y Tatiana Sandoval

\section{Resumen}

El presente artículo examina el interesante caso de pobladores andinos longevos que se sitúan en los estratos de pobreza extrema en el Perú. Se usa el término "longevidad" para referirse a cualquier individuo que tiene más de noventa años y que es activamente funcional. Existen escasas referencias antropológicas sobre la longevidad en los andes peruanos. Aquí nos enfocamos en las personas que alcanzan los cien años o más en condiciones de extrema pobreza y a quienes pudimos visitar en el campo, en donde nos contaron sus modos de vida y lo que significa para ellos llegar a tan avanzada edad y cómo perciben su propia longevidad. Para ello, se tomaron en cuenta diversos factores como la alimentación, las relaciones sociales y las creencias de estas personas.

\section{Palabras clave}

Longevidad, centenarios, salud, extrema pobreza, Andes. 


\title{
Longevity and extreme poverty in Huancavelica, Perú
}

- Úrsula Sandoval y Tatiana Sandoval

\begin{abstract}
This article analyzes the case of local Andean long-lived population that paradoxically are located in extreme poverty areas in Peru. The term "longevity" is used to refer to any individual who is over ninety years of age and who is actively functional. There are only a few anthropological studies on longevity in the Peruvian Andes. This study focuses on people that reach an age of one hundred years or more living in extreme poverty conditions and who we could visit during fieldwork. The interviewees explain their ways of life and what it meant to reach such an old age and how they perceived their own longevity. For this matter, many diverse factors were taken into account, such as feeding, social relationships and personal beliefs.
\end{abstract}

\section{Keywords}

Longevity, centenarians, health, extreme poverty, Andes. 


\section{Introducción}

Nuestro trabajo de campo lo llevamos a cabo en el año 2016 en el mes de julio. Nos enfocamos en la provincia de Huancavelica, en especial en el distrito de Acoria, ya que se nos informó sobre la existencia de varios longevos en esta zona. También visitamos otros distritos y comunidades campesinas. Un poco más de la mitad de los centenarios pobres del Perú se encuentran viviendo en los Andes y muy especialmente en los Andes Centrales. ¿Qué tiene de especial esta zona en particular para que las personas que viven allí pasen los cien años de edad? Según Pensión 65, el 55\% de los centenarios empadronados por este programa se encuentran en Ayacucho, Huancavelica, Apurímac y, también, Puno (Ministerio de Desarrollo e Inclusión Social, 2014).

Tener cien años en una provincia del Perú no es lo mismo que tenerlos en la capital, Lima. Menos aún es tenerlos en un país del primer mundo. Las estadísticas muestran que la mayor cantidad de población centenaria se encuentra en los países desarrollados debido a que tienen mejores condiciones de vida y fácil acceso a los servicios de salud. El Instituto Max Planck en su estudio demográfico Supercentenarians (2010) señaló que los Estados Unidos alberga el mayor número de supercentenarios, siguiéndole Japón, Inglaterra, Gales, Francia e Italia. Aquí en el Perú se conocen algunos casos de supercentenarios, pero estos no han sido validados por el Gerontology Research Group ${ }^{1}$.

\section{Antecedentes históricos:}

Sobre la salud de los centenarios en el Perú, las ciencias sociales han escrito muy poco y, por lo general, se ha hecho casi de manera anecdótica. Desde una perspectiva histórica sabemos que ciertas crónicas coloniales mencionan que existieron centenarios en el Perú a la llegada de los españoles. En el siglo XVII, el cronista español Bernabé Cobo en su en su crónica Historia del Nuevo Mundo, refiriéndose al altiplano, escribe:

[..]Es la parte más sana del Perú y donde viven muchos hombres, en ningunaparte de este reino he visto menos enfermos ni mayor número de indios viejos de más de 100 años que se acordaban del tiempo de los reyes incas y de la entrada de los españoles [...] todos tienen buena dentadura y $\tan$ recia que les dura toda la vida...raras veces padecen de dolor de muelas ni corrimiento en ellas, y es raro el indio viejo a quien falta la dentadura. (Cobo,1580-1653, p. 55-57)

Otra crónica, la de Huamán Poma de Ayala, menciona a Mama Cora Ocllo, mujer del Inca Lloque Yupanqui que murió en el Cusco a la edad de 120 años. De la misma edad murió la coya de Inca Roca, Mama Michay. Muchas de las coyas descritas por Huamán Poma pasaban los cien años.

1. El Gerontology Research Group se encarga de verificar y validar la edad de las personas supercentenarias en el mundo. 
Otro dato histórico lo encontramos en una carta publicada en el periódico Mercurio Peruano en el año 1792 firmada por Q.S.M.B Chiros-Atychio Presbyógrapho desde Chota, Cajamarca. La carta está dedicada exclusivamente a la longevidad de ciertos habitantes del Perú. En ella se mencionan a varias personas con edades asombrosas de 135 y 147 años. La persona que escribe la carta evidentemente no muestra ningún documento que pruebe las edades de las personas a las que hace mención. Sin embargo, hace referencia a una partida bautismal que él mismo pudo observar de una mujer de 131 años que vivió en el distrito de Polloc en la provincia de Cajatambo, departamento de Cajamarca. Según el autor todo lo que vio fue corroborado por otros vecinos que daban por verdad todos estos hechos.

Más recientemente, en 2014 hubo un caso muy sonado, el de Filomena Taipe, proveniente del distrito de Acoria en Huancavelica que alcanzó la edad de 117 años. Su documento de identidad muestra que nació el 20 de diciembre de 1897 . Su notable longevidad mereció varios titulares en la prensa alrededor del mundo. Se hizo conocida gracias a Pensión 65, un programa del Ministerio de Desarrollo e Inclusión Social del Estado peruano que otorga una subvención económica de 250 soles bimestrales a los adultos mayores a partir de los 65 años de edad que viven en condiciones de extrema pobreza. Desde su propia perspectiva, Filomena contaba que su longevidad se debía a la alimentación natural y a los productos de la chacra. Se alimentaba de papa, oca, mashua, carne de cabra y carnero, leche, queso de cabra y habas, no consumía alimentos enlatados ni procesados, tampoco gaseosas. Según José Francisco Parodi, médico geriatra experto en envejecimiento y longevidad, responsable del Centro de Investigación del Envejecimiento de la Universidad San Martín de Porres, Filomena Taipe es un caso de supervivencia extraordinaria; señala además que estos longevos descienden de familias longevas y que la alimentación del campo les ha ayudado a prevenir enfermedades.

\section{Validación de las edades de personas centenarias en otras partes del mundo}

Es importante mencionar los requisitos que se tienen que cumplir para tener certeza sobre las edades de los centenarios. Según el Gerontology Research Group, para hacer fidedigna la longevidad de una persona se necesita contar con al menos tres documentos, entre los que se incluyen el acta de nacimiento otorgado por el gobierno, un certificado bautismal, censos, y/o un certificado de matrimonio que muestre el cambio de nombre de la mujer. Japón, por ejemplo, es uno de los países que concentra gran cantidad de longevos en el mundo, en donde cada ciudad, villa y pueblo cuenta desde 1879 con un sistema de registro familiar llamado kosek ${ }^{1}$, gracias al cual se pueden verificar los datos de nacimiento, paternidad, adopción matrimonios y muertes de todos los ciudadanos japoneses. Al ser uno de los registros más antiguos del mundo, no quedan dudas de los documentos que prueban la elevada cantidad de centenarios.

El caso de los Andes peruanos es distinto. Debido a diversos factores, nuestro país en muchos casos no cuenta con los registros oficiales de datos necesarios para comprobar si una zona goza de longevidad; y los pocos registros que hay en provincias se han perdido a causa de incendios, desapareciendo de esta manera mucho material valioso. 


\section{Distinciones sociológicas de la longevidad.}

Hay que distinguir entre lo que se conoce como "longevidad individual" y "longevidad poblacional". Existen varios casos de longevidad de individuos que han sobrepasado los 115 años. Uno delos casos más sorprendentes en el mundo fue el de Jeanne Calment, la francesa que, de acuerdo al Libro Guinness, aún ostenta el título de la persona que vivió más tiempo en toda la historia, al menos, según registros comprobados. Calment murió en 1997 a los 122 años y 164 días de edad. La longevidad poblacional en cambio, se trata de una zona geográfica, que por lo general no es muy grande y concentra una importante cantidad de longevos y centenarios. Un ejemplo de esto son las denominadas “Blue Zones” o Zonas Azules. Según Michel Poulain, demógrafo, experto en estudios sobre longevidad y coautor del libro The Blue Zones (2013), este término hace referencia a un área geográfica bastante pequeña y homogénea, en donde la población comparte el mismo estilo de vida y el mismo medio ambiente y su longevidad excepcional ha sido científicamente probada. Poulain agrega también que por lo general, las poblaciones de una Blue Zone se han mantenido geográfica y/o históricamente aisladas, en islas o regiones montañosas. Estas poblaciones han conservado un estilo de vida tradicional y muchos han seguido una intensa actividad física que se extiende más allá de los 80 años. También el consumo de comida local y el soporte de la comunidad se han considerado como posibles causas de la longevidad de estas personas. Se han identificado 4 regiones en el mundo que poseen los requisitos para alcanzar el status de Zona Azul: 1) Ogliastra, Cerdeña, Italia. 2) Okinawa, Japón. 3) La Península de Nicoya, Costa Rica. 4) Ikaria, Grecia.

En estudios internacionales sobre longevidad, los investigadores advierten que muchas personas alteraron sus edades. Es el caso, por ejemplo, del valle de Hunza en Pakistán. En este lugar, muchos longevos afirmaban tener cierta cantidad de años, incluso apoyados por el mismo gobierno, pero cuando se hizo la investigación correspondiente no se pudo encontrar documentos que apoyen estas afirmaciones. Lo mismo ocurrió en el valle de Vilcabamba en Ecuador. Por lo tanto, para saber si un área geográfica es excepcional en longevidad se tiene que hacer una investigación interdisciplinaria, usando la metodología de la demografía, la historia y la antropología.

\section{La longevidad en Huancavelica en estudio}

Nuestros objetivos fueron dos: verificar en el campo la presencia de personas centenarias, y mostrar cómo es percibida la longevidad y la salud en condiciones de extrema pobreza por los propios centenarios.

Nuestra metodología consistió en la elaboración de una serie de preguntas sobre los estilos de vida de estas personas. Esta guía de preguntas que en el campo se plasmó como "entrevista no estructurada" nos permitió hacer un trabajo flexible

1. Según la investigación de los hermanos Willcox (2001): "Koseki es un sistema nacional de registro de hogares. Según el sistema, cada hogar japonés tiene su registro, el cual mantiene los nombres de los padres que son cabeza de familia y de todos los miembros de la familia”. De acuerdo a los hermanos Willcox, Koseki es una de las fuentes más antiguas y confiables de información sobre muertes y otras estadísticas de población en el mundo. Ha estado funcionando en Okinawa desde 1879 y en Japón desde 1871. 
pero al mismo tiempo controlado, logrando que el entrevistado pueda relatar sus experiencias y percepciones de manera natural y libre. Todos nuestros informantes eran quechua hablantes. Ninguno hablaba el castellano. Nuestro informante Juan Ccanto fue el que hizo de traductor en algunos casos. Los hijos y nietos de los centenarios también participaron en la traducción.

Decidimos indagar sobre el estilo de vida tratando de obtener una visión completa sobre alimentación, enfermedades, ejercicio, creencias, relaciones sociales, conocimiento tradicional y clima. Descubrimos que muchos de estos centenarios tienen percepciones muy parecidas sobre su longevidad. Es posible que el mismo ambiente cultural les haya otorgado una visión similar; sin embargo, también encontramos que cada uno maneja un discurso muy propio y subjetivo sobre sus años; así encontramos, por ejemplo, que para algunos es una bendición ser tan longevo y para otros una maldición. Privilegiamos en este primer trabajo de campo las entrevistas personales a los longevos y a los centenarios en su mismo entorno. Durante las semanas de permanencia en Huancavelica ubicamos a los informantes para registrar la localización exacta de cada longevo y centenario; conversamos con ellos, verificamos sus datos en el DNI respectivo y tomamos fotos de cada una de estas personas. Por lo general, desplazarse requería dos horas de la ciudad de Huancavelica al centro poblado o comunidad en la que habitan los longevos, lo que hacía que una parte importante del tiempo la pasemos en la ruta hacia su encuentro. Queremos destacar que hemos contado con los datos proporcionados por Pensión 65. El Jefe de Pensión 65 de Huancavelica, Yackson Ynga, señaló que a los centenarios se les otorga el documento de identidad (DNI) en base a los registros que la RENIEC posee.

\section{La longevidad en sus datos empíricos}

La tabla 1 resume los datos de nuestros informantes centenarios que viven en el distrito de Acoria, proporcionada por Pensión 65 de Huancavelica:

\begin{tabular}{|ccccccccc|}
\hline DNI & $\begin{array}{c}\text { Apellido } \\
\text { Paterno }\end{array}$ & $\begin{array}{c}\text { Apellido } \\
\text { Materno }\end{array}$ & Nombres & $\begin{array}{c}\text { Fecha de } \\
\text { Nacimiento }\end{array}$ & Edad & Distrito & Comunidad \\
\hline 48060596 & Díaz & Janampa & Marcelino & $02-02-1913$ & 103 & Acoria & Ccaccasiri \\
20178709 & Huamán & De Paucar & Victoriana & $10-10-1916$ & 100 & Acoria & Motoy Pata \\
80231565 & Ticsihua & Carhuapoma & Dionisio & $24-06-1912$ & 104 & Acoria & Patahuasi \\
23255069 & Taipe & Soto & Encarnación & $25-03-1994$ & 102 & Acoria & Ruruncancha \\
47003408 & Tuncar & Cárdenas & Ricardina & $10-01-1926$ & 100 & Huancavelica & Ccaccasiri \\
48132052 & Castro & Mendoza & Saturnina & $20-02-1908$ & 108 & Acoria & Ccaccasiri \\
23221809 & Benito & Sedano & David & $31-03-1913$ & 103 & Acoria & Acoria \\
48481188 & Quispe & Quincho & Faustina & $03-06-1911$ & 115 & Acoria & Acoria \\
23237048 & Quispe & Villaba & Patricia & $12-08-1912$ & 104 & Acoria & Acoria \\
\hline
\end{tabular}


De los nueve centenarios presentados en esta tabla, pudimos acceder a entrevistar a tres. Entrevistamos también a nonagenarios de otras comunidades que dieron su percepción sobre la longevidad, todos en condiciones de extrema pobreza. De igual manera creemos oportuno compartir la percepción de un centenario que migró de Huancavelica a Lima. Si bien ya no vive en condiciones de extrema pobreza, es importante su testimonio porque da cuenta que, a pesar de la migración, trató de mantener su estilo de vida, especialmente el concerniente a la alimentación.

Al encontrar percepciones muy parecidas entre los entrevistados, decidimos organizar la información de acuerdo a los principales temas que rodean la longevidad: alimentación, enfermedades en los centenarios, ejercicio y relaciones sociales.

Estilos de Alimentación: Según los datos arrojados en las entrevistas, los longevos desde el inicio de sus vidas se alimentaron comiendo productos propios de la chacra como mashua, oca, papa, chuño, olluco, haba, cebada, trigo, maíz, y de los animales que criaban, tales como alpacas, carneros y cabras. En aquellos tiempos no se utilizaban fertilizantes químicos en la agricultura, incluso, contaban que la gente sembraba sin guano, lo que denota la gran fertilidad que la tierra tenía. En la actualidad, como dijo el alcalde del centro poblado de Huayllayocc, T.P, ahora se necesitan reactivar los cultivos andinos con tecnificaciones y maquinarias modernas para que puedan seguir creciendo los alimentos y capacitar a los campesinos ya que estos han dejado de trabajar en la chacra como antes. De manera similar a la opinión del alcalde, nuestro informante J. C (40) mencionaba: "Anteriormente full chacrita, el clima ha cambiado, pero también ahora nos hemos vuelto ociosos, chacra ya no tiene nadie".

Todos los entrevistados manifestaron que se comía de manera natural y recalcaron que los fideos, el atún, el aceite, son artificiales y muy procesados: "Todo era chacra antes, se comía morón, habas, mashua. Fideo no comíamos, de eso poco, mamá”, (mujer de 78 años que acompañaba y traducía a longeva de 96 años). Lo mismo nos dijo para la leche: "De las vacas sacábamos, pero eso era anteriormente, ahora es de tarro nomá". Juan, nuestro informante agregaba: "Comían chuño, trigo, kiwicha, quinua, todo, no había fideos, no había azúcar, también era poco, eso es malo, o sea, la comida ya es bien procesada actualmente". Mario del Río, centenario de 103 años, en excelentes condiciones de salud, migrante de Huancavelica, actualmente viviendo en Lima, cuenta sobre la alimentación:

Todo lo que hay ahora, eso no comía, yo no conocía fideo, atún, leche, yo conocía eso cuando era ya muchacho. Cuando mi papá venía y yo tenía 12013 años, me llevaba de la sierra a la costa, allí ya comía fideos que parecía tripa de vaca, me daba asco, comía fideos de todo tamaño, largo, redonditos, yo me preguntaba ¿̇qué cosa es esto?, pero no le encontraba gusto, sin embargo, no me caía mal, estaba joven, pues. Mi mamá me daba mucha cebada molida con batán, yo tengo un batán que me he traído de mi sierra para que chanque charqui. El charqui asado en fogón, eso comía antes, bueno, yo comía eso porque en la sierra no había azúcar, arroz. Íbamos a Cañete, tres días de viaje y otros 3 días de regreso y traía azucarcito y salcito. Antes, entre Huancavelica y Huancayo había sal de piedra y teníamos que remojar en agua y el agua la ponías al caldo a cualquier comida. 
Simeona, hija de Patricia Quispe, longeva de 104 años, dice:

Comían sopa phatasqa (patasca) con aceros, hacían hervir con acero dice mi mamá, comía su cancha con su fiambre, con cancha de maíz, con queso de cabra. Mis abuelos me decían que para qué comer fideos, arroz, pollo; por eso mi hermana también me decía que yo parezco más vieja que ella, mi hermana siendo mayor que yo no come de eso y se ve más joven.

Observamos además, que por lo general, se cocina con leña y bosta. De todas las visitas que realizamos, sólo la centenaria de 104 años P.Q. ya cocinaba con cocina mejorada; el hecho de haber cocinado mucho tiempo en sus fogones rústicos le produjo enfermedad de las vías respiratorias y una tos constante con flema, esto debido a la presencia de humo y su inhalación constante. La cocina mejorada le significó un ahorro de leña y menos humo; sin embargo, las otras cocinas que observamos seguían conservando su forma tradicional, lo cual convierte al ambiente en un entorno no propicio para la salud familiar debido a la presencia de dicho humo.

A pesar de que los longevos y centenarios consumen en mayor medida una alimentación natural, mencionaron que mucho de lo que se consume hoy en día es una alimentación combinada, esto es, una alimentación natural junto con una alimentación procesada ${ }^{1}$. Cuando se le preguntó a nuestro informante Juan que tradujera lo que come B.P. en la actualidad, longeva de 96 años en buen estado de salud, dijo lo siguiente: "Pan, fideos, de todo un poco ahora, cuando quiere beber algo va a la tiendita de al lado y toma su frugos y su yogurt; aunque ella misma generalmente se cocina sus propios alimentos". Al preguntarle a nuestro informante si él come comida natural y si sus hijos también lo hacen, nos respondió lo siguiente: "Combinado, a mis hijos le doy ya todo combinado... es que ya no se encuentra tan fácil" (refiriéndose a la comida natural), "en las tiendas ves poco, poquito chuñito, y es la gente mismo que despreciamos las ricas comidas; los niños más que nada ya no quieren comer chuño".

Ejercicio y desplazamiento físico: Las personas más longevas del mundo viven en entornos que les inducen a moverse naturalmente. Los Andes, al ser un entorno montañoso, propician que la gente camine mucho. Hemos observado a personas ancianas caminando cuesta arriba, cuesta abajo y casi siempre cargando algo sobre sus espaldas. Casi toda la vida de estos centenarios estuvo marcada por el trabajo en la chacra, por traer y llevar mercancía a distancias lejanas, llevando peso a cuestas. Las condiciones de pobreza han obligado a estas personas a caminar para traer alimento y agua desde que eran niños. Se han acostumbrado a caminar toda su vida. Lo que vimos en el campo fue a ciertos longevos y centenarios desplazarse con relativa facilidad, apoyándose por lo general de un bastón.

Cuando se le preguntó a M.R, centenario de 103 años, originario de Huancavelica residente en Lima, sobre si realizaba algún tipo de actividad física en la actualidad, comentó: "Sí, camino, ando mucho, en mi cama también hago movimientos, si no me muevo el cuerpo se me tiembla". Desde su casa en el cercado de Lima, va caminando hasta el mercado de la Parada para comprar hojas de coca, va solo sin la compañía de nadie, se apoya en un bastón y a pesar de tener ceguera en un ojo nada le impide ir al mercado a comprar su coca: "Conozco el camino y sé por dónde ir". De igual 
manera la madre de nuestro informante Juan Ccanto, de 96 años, cuenta lo que piensa acerca del ejercicio: "Camino, camino mucho, si no camino los pies empiezan a encogerse, y cuando camino, los pies están bien sueltos”.

Actualmente, todos los longevos que visitamos podían caminar solos, siempre con la ayuda de un bastón. El nieto de la longeva de 104 años cuenta sobre su abuela: "Ella está sana, sólo su tos, camina muy bien, va solita hasta la posta, hasta abajo, nadie la lleva”. La supercentenaria de 115 años, F.Q. fue la única excepción, ella tenía las piernas inmóviles y dependía completamente de su hija incluso hasta para vestirse. Ella hasta los 112 años caminaba de vez en cuando, ahora ya no puede. Los longevos de los Andes, si bien ya no pueden trabajar como lo hacían antes en la chacra, eso no les ha impedido moverse con facilidad. Cuando caminan es para ir, por ejemplo, a una junta en la comunidad o para ir a la posta, también cuando van a comprar o incluso a vender en el mercado, y lo hacen casi siempre sin compañía. Podemos decir que los longevos y centenarios viven una vida de relativa actividad e independencia.

Enfermedades comunes en los longevos y centenarios: Queremos subrayar que al momento de hacer nuestro trabajo de campo, el jefe de Pensión 65 nos comentó que Huancavelica no cuenta con ningún médico geriatra. Se nos dijo también que los médicos que se ocupan de estos ancianos son, por lo general, practicantes.

$\mathrm{Al}$ entrevistar a Y.Y, Jefe del programa Pensión 65 de Huancavelica, le preguntamos cuáles son las enfermedades más comunes en el adulto mayor. Nos contestó lo siguiente:

Según la Dirección Regional de Salud, la DIRESA, la enfermedad que prevalece es el cáncer al estómago, pero generalmente lo padecen los abuelitos que viven en las ciudades, muchas veces abandonados y que tienen mucha dependencia de la economía, entonces cuando no tienen dinero no pueden comer y cuando comen por lo general es comida chatarra, lo cual va a degradar su sistema digestivo.

Queremos añadir a esto, que el médico oncólogo R.V., director médico de la Liga Contra el Cáncer, sostuvo que es en la sierra donde el cáncer de estómago se presenta como el más común por una forma de comer que viene de épocas incaicas: "Son las carnes que las ponen a la intemperie, les echan sales y las hacen secar, lo que nosotros conocemos como el charqui”. La Organización Mundial de Salud (OMS) ha advertido a través de un comunicado en el año 2015 que hay suficiente evidencia para relacionar la charcutería con el cáncer de colon. El consumo de cualquier tipo de carne que sea procesada, mediante de la salazón, el curado, la fermentación o el ahumado podría inducir al cáncer. La OMS no menciona explícitamente al charqui, pero de acuerdo a los médicos peruanos este cabe en la denominación de carne procesada.

Las Relaciones Sociales: Si bien la alimentación, el agua, el ejercicio son factores claves en el proceso de envejecimiento saludable, subrayamos aquí la importancia, incluso mayor, de las relaciones sociales. Nuestra conversación con el Jefe de Pensión 65, Y.Y, sobre la situación del adulto mayor en Huancavelica mostró una faceta

1. Por alimento procesado se entiende que es todo alimento que el hombre ha transformado mediante procesos industriales, mecánicos y químicos para alargar y conservar la vida en el empaque. 
que, al menos en el Perú se ha descuidado mucho. A continuación reproducimos textualmente lo que nos dijo:

La red social del adulto mayor se quiebra cuando es adulto mayor. Se rompe, esté en familia o esté solo. Un adulto mayor quiere ser responsable de él mismo y quiere dejar de ser carga para su familia y en el peor de los casos, ser abandonado por los hijos. Migran a Lima y se olvidan de los papás: "cómo vivirá mi papá... no sé, pero debe estar bien en su pueblo", entonces los abandonan, el abuelito ya no tiene fuerzas. Ustedes saben, en la zona andina existe el tema de la comunidad, pero la comunidad, las comunidades campesinas también se vienen degradando con el pasar de los años. Anteriormente teníamos a los abuelitos como los sabios, como quienes guiaban, eran quienes estaban a la cabeza y tenían su consejo de ancianos para tomar decisiones sobre ciertas actividades que se realizaban. En la actualidad, las comunidades campesinas ya no funcionan así. Quienes han tomado la batuta son los que tienen la fuerza, que son los jóvenes que no saben nada, que no tienen experiencia y a los abuelitos los han relegado, ¿̇por qué?, porque en esta sociedad la fuerza es importante y quienes participan de una comunidad son los que pueden hacer ayni. Lo que hace la sociedad es obligarlos a morir y es lo que va a pasar con nosotros también. En los países del primer mundo, los abuelitos tendrán mejores condiciones de vida, acceso a la salud, pero están igual de abandonados y la tristeza es lo que psicológicamente te mata. ¿Qué pasa con estos centenarios? Los centenarios dicen muchas veces que lo único que quieren es que Dios los lleve, sobre todo los que están abandonados o viven solos. Eso es que lo generalmente dicen porque no se sienten bien, no son lo mismo, porque su capacidad, su calidad de vida, su economía y su entorno social entre otras cosas, se ha quebrado. Entonces, el entorno social es muy importante. Lo que hacemos nosotros es decirle a la autoridad local que tienen que trabajar con adultos mayores.

\section{Conclusiones}

Nos enfocamos en las visitas a longevos y centenarios que viven en comunidades alejadas de las ciudades. El entorno natural de alguna manera les es propicio para llevar un ritmo de vida menos estresado y acelerado. El contacto con zzvtel hecho de vivir en una zona de montañas es posible que haya influido en la longevidad de estas personas fortaleciendo su biología. Creemos posible que el estilo de vida que llevaron desde pequeños, siendo éste más natural, ha ayudado a su sistema inmunológico a combatir muchas enfermedades consideradas graves y de las que ahora cada vez más los jóvenes padecen.

Si bien es cierto que viven en zonas consideradas de extrema pobreza, ya que no cuentan con los servicios básicos de agua potable ni servicios de salud, no ha impedido que puedan llegar a tener una extrema longevidad. Con esto queremos decir que han sabido utilizar de manera eficiente sus propios recursos o lo que les proporcionaba su entorno, y a través de sus conocimientos tradicionales aprovechar lo que la naturaleza les proveía. Tal vez sea la última vez que veamos a una generación de longevos y centenarios hacer uso de su conocimiento tradicional ya que los hijos de estos longevos están completamente inmersos en la cultura moderna de las ciudades. Hemos querido aquí dar cuenta de que la pobreza no excluye la longevidad. Es cierto que en los países del primer mundo la cantidad de longevos y centenarios comprobados es mayor que en los Andes. También la calidad de vida es superior a 
la de las comunidades altoandinas; sin embargo, existe una población de longevos y centenarios andinos que tiene algo que contar sobre la longevidad. De todas maneras, consideramos importante continuar estudiando el fenómeno de la longevidad en condiciones de extrema pobreza ya que puede aportar en gran medida al proceso de envejecimiento. Los factores genéticos son muy importantes, pero las últimas investigaciones están considerando que los factores epigenéticos y el estilo de vida son determinantes en la forma de cómo uno envejece. Nuestro estudio ha querido ofrecer una mirada antropológica y mostrar cómo se vive la longevidad en extrema pobreza en el Perú. Hemos empezado por la provincia de Huancavelica y eventualmente haremos un estudio comparativo con otras provincias andinas para resaltar las diferencias y similitudes en los factores que propician la longevidad en los Andes del Perú.

Sugerimos que en un futuro próximo se profundice en la validación de las edades de los centenarios. Se requiere hacer una búsqueda en archivos para encontrar partidas de nacimiento, bautizo o matrimonio en donde se compruebe que estas personas son efectivamente centenarias. Según los centros internacionales de investigación sobre longevidad, el DNI no basta para cumplir con los criterios de validación de edades. Otra línea de estudio que consideramos se desprende de esta investigación es la elaboración de un análisis comparativo sobre el estilo de vida de los longevos y centenarios cuando eran jóvenes con la de los jóvenes en la actualidad, lo cual podría arrojar datos interesantes sobre sus modos de vida y así determinar qué población pudiera estar relativamente más sana. 


\section{BIBLIOGRAFÍA}

Antúnez de Mayolo, S. (1981).

La Nutrición en el Antiguo Perú. Lima, Perú: Banco Central de Reserva del Perú.

Buettner, D. (2008).

The Blue Zones. 9 lessons for living longer from the people who've lived the longest. Washington, D.C: National Geographic Society.

Cobo, B. (1580-1653).

Historia del Nuevo Mundo. Madrid, España: Artes Gráficas 1956.

Del Pino, P.; Mena, M.; Torrejón, S.; Del Pino, E.; Aronés, M.; Portugal, T. (2012).

Repensar la desnutrición. Infancia, alimentación y cultura en Ayacucho, Perú. Lima, Perú. Instituto de Estudios Peruanos.

Demographic Research Monographs 7. (2010).

Supercentenarians. Berlin, Germany: Springer.

Gerontology Research Group.

Base de datos de los supercentenarios del mundo del Gerontology Research Group. Recuperado de: http://www.grg.org/SC/SCindex.html

Base de datos de los supercentenarios del mundo del Gerontology Research Group. Recuperado de: http://www.grg.org/SC/SCindex.html

Guamán Poma de Ayala, F. (1615/1616).

El Primer Nueva Corónica y Buen Gobierno.

Holmes, E.; Holmes, L. (1995).

Other Cultures, Elder Years. California: Sage Publications, Inc.

Le, S. (2016).

100 Million Years of Food. What our ancestors ate and why it matters today. New York City:

Picador.

Mercurio Peruano. (1861).

Tomo Tercero. Lima, Perú.

Ministerio de Desarrollo e Inclusión Social. (2014).

55\% de centenarios de Pensión 65 está en Ayacucho, Apurímac, Huancavelica y Puno. Pensión 65. Recuperado de: http://www.pension65.gob.pe/2014/05/55-de-centenarios-de-pension65-estan-en-ayacucho-apurimac-huancavelica-y-puno/

Poulain, M.;Herm, A.; Pes, G. (2013).

The Blue Zones: áreas of exceptional longevity around the world. Vienna Yearbook of Population Research, vol. 11.

Ramos, G. (2014).

iAquí nadie es viejo! Usos e interpretaciones del Programa Centro del Adulto Mayor- EsSalud de Villa María del Triunfo y las experiencias de envejecimiento de sus usuarios. Tesis para optar el Título de Licenciada en Antropología. Lima, Perú: PUCP. 
Simon, Stacy (2015, 6 de octubre).

World Health Organization says meat causes cancer. Recuperado de: https://www.cancer. org/latest-news/world-health-organization-says-processed-meat-causes-cancer.html

Ulloa, A. (1772).

Noticias americanas: entretenimientos phísicos-históricos sobre la América Meridional y la Septentrional Oriental. Madrid, España: Imprenta de don Francisco Manuel de Mena.

Willcox, B.; Willcox, C.; Suzuki, M. (2001).

The Okinawa Program. Learn the secrets to healthy longevity. New York City: Three Rivers Press. 